\title{
LA GESTION DES TERRES PUBLIQUES ET SES CONSÉQUENCES SUR LE PATRIMOINE FORESTIER \\ Josy MAZODIER
}

\section{LES TERRES PUBLIQUES}

\section{Leurs origines}

Avant la création de la Confédération canadienne, la Couronne britannique était propriétaire de toutes les terres qui n'avaient pas été érigées en seigneurie par le Roi de France ou données en concession après la conquête anglaise.

"L'Acte de l'Amérique du Nord britannique " qui, en 1867 marqua la naissance du Dominion donne aux provinces la propriété de l'usage de ces terres, à l'exception de celles attribuées nommément à la Confédération.

\section{Leur nature physique et leur répartition}

Les terres publiques couvrent $1450000 \mathrm{~km}^{2}$, soit $91,5 \%$ du territoire du Québec.

En raison même de leur origine, elles sont inégalement réparties sur le territoire de la Province. Elles ne représentent qu'environ le tiers de l'espace dans les régions riveraines du Saint-Laurent qui ont été colonisées les premières (Québec de base), alors qu'elles couvrent la quasi totalité des régions les plus excentriques (Nouveau Québec, Côte Nord, etc.).

Elles peuvent être divisées succintement en trois zones. Au nord du $58^{\mathrm{e}}$ parallèle, sur près de $300000 \mathrm{~km}^{2}$ s'étend la " toundra " où marais, lichens et peuplements arbustifs se mêlent. Entre le $52^{\circ}$ et le $58^{\ominus}$ parallèle, on rencontre la "taïga " qui se caractérise par ses peuplements clairs de bouleaux et de résineux. Ces deux premières zones, du fait de leur éloignement et de la rigueur des conditions de vie ne présentent actuellement que peu d'intérêt économique, à l'exception de certains gisements de minerais (cuivre). En revanche leur richesse écologique est tout à fait remarquable. Au sud du $52^{\mathrm{e}}$ parallèle, la forêt dite commerciale, sapinière et pessière au nord et érablière au sud, couvre $613000 \mathrm{~km}^{2}$.

Cette dernière zone contient l'essentiel des ressources naturelles susceptibles d'une mise en valeur dans les conditions économiques actuelles: minerais, bois, énergie hydraulique, paysages, ressources halieutiques et cynégétiques, etc. C'est également celle qui présente la plus grande valeur stratégique du point de vue du développement, car s'y inscrivent nécessairement toutes les activités en rapport avec l'utilisation de ces ressources. 


\section{Leur statut juridique}

Depuis 1867 , le statut n'en a guère changé. Le caractère de possession de la Couronne britannique survit encore sous la forme de la tutelle, théoriquement absolue, qu'exerce le lieutenantgouverneur en Conseil qui la représente. Mais le rôle de ce haut fonctionnaire de la Couronne est très effacé, et, dans la pratique, l'usage de ces terres est totalement placé sous l'autorité du gouvernement provincial.

Toutes les terres sur lesquelles ni l'État fédéral, ni les collectivités locales, ni les particuliers ne peuvent se prévaloir d'un droit de propriété, sont considérées comme terres publiques. Comme l'ensemble des biens immobiliers de la Province, elles sont selon le droit strict, imprescriptibles, inaliénables, insaisissables et ne sont pas soumises à l'impôt foncier. Néanmoins, pour permettre leur mise en valeur, le principe d'inaliénabilité est tombé en désuétude dans l'usage administratif. Elles peuvent donc être amodiées ou aliénées selon des formules nombreuses : lettre patente, permis d'exploitation ou d'occupation, location, bail, concession ou vente.

\section{LA GESTION DES TERRES PUBLIQUES}

Le Gouvernement du Québec a la maîtrise complète de I'utilisation des terres publiques. II lui revient d'inventorier les richesses de ce patrimoine, de les protéger, d'en planifier et d'en contrôler la mise en valeur, de pourvoir aux besoins en espace des différents agents économiques et des collectivités locales, mais aussi de répondre aux aspirations croissantes du public à pouvoir disposer d'espaces de nature et de récréation.

\section{Organisation administrative}

Plusieurs ministères se partagent, selon les compétences sectorielles que leur donne la loi, l'administration des terres publiques. Ils peuvent être chargés de la mise en valeur et de la protection de certaines ressources naturelles sur toutes ces terres, ou appliquer des règles particulières de gestion sur les territoires qui sont placés sous leur juridiction.

Quatre ministères détiennent un rôle important : le ministère des Terres et Forêts, le ministère de l'Agriculture, le ministère des Richesses naturelles et le ministère du Tourisme, de la Chasse et de la Pêche.

\section{Le ministère des Terres et Forêts}

Le ministère des Terres et Forêts est responsable de la gestion foncière des terres publiques. A ce titre:

- il est chargé de la tenue du "Terrier ", registre officiel sur lequel sont consignés les renseignements relatifs à la localisation, la délimitation, la superficie des terres publiques et privées, ainsi que les droits et servitudes s'y rapportant ;

- il a autorité pour amodier ou vendre les terres publiques ou les placer sous la juridiction un autre ministère, d'un organisme public ou para-public ou même du Gouvernement fédéral;

- il procède à l'arpentage et à la cartographie des biens immobiliers de la province ;

- il surveille les terres publiques et intervient contre les occupations illégales.

Le Ministère est également chargé de la ressource ligneuse dont il assure :

- la conservation en organisant la défense contre les incendies et les interventions phytosanitaires par l'intermédiaire de sociétés de conservation, en veillant à la répression des coupes abusives et à l'information du public ; 


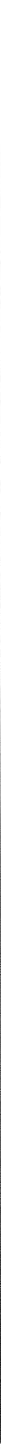
Photo Éditeur officiel du Québec

- la mise en valeur par la délivrance d'autorisation de coupe.

\section{Le ministère du Tourisme, de la Chasse et de la Pêche}

II est chargé de la protection et de la mise en valeur des ressources cynégétiques et halieutiques, et d'une manière générale de l'utilisation touristique des terres publiques.

Il lui incombe à ce titre :

- de définir les parties du territoire public sur lesquelles peuvent être délivrés des droits de chasse et de pêche; 
- de délivrer des "permis de pourvoyeurs" (organisation à but lucratif d'expéditions de chasse et de pêche);

- de créer et gérer des réserves de chasse et de pêche dans lesquelles ces activités sont soumises à des règles particulières;

- de créer et de gérer des parcs provinciaux.

De plus, ce Ministère assure directement la conception, la création et la gestion de certains équipements touristiques (campings, stades de neige, etc.) ou de certains centres destinés à sensibiliser le public sur le milieu naturel.

\section{Le ministère de l'Agriculture}

II doit déterminer les parties du territoire public qui offrent des conditions favorables à une mise en valeur agricole. II définit les programmes de "colonisation ", c'est-à-dire de lotissements des terrains à ouvrir à l'agriculture, et effectue conjointement avec le ministère des Terres et Forêts les opérations foncières correspondantes.

\section{Le ministère des Richesses naturelles (1)}

Il est responsable de l'exploitation des ressources minérales et énergétiques et de la gestion du régime des eaux.

Il délivre les permis de prospection minière, les autorisations d'effectuer sur les terres publiques des travaux de recherche et d'y construire les installations nécessaires (claims miniers). Les terrains devant faire l'objet d'une exploitation minière sont ensuite cédés en pleine propriété.

Ce ministère autorise les occupations sur les cours d'eau navigables et flottables ainsi que les travaux et les prélèvements de matériaux qui y sont effectués. Il assure le contrôle des barrages, du flottage des bois et de tous les ouvrages qui se rapportent à cette activité.

La Société d'État "Hydro-Québec " détient le monopole de la mise en valeur du potentiel hydroélectrique. Elle possède les barrages et les lignes électriques et peut obtenir la propriété des terrains qui lui sont nécessaires (lignes électriques, etc.) ainsi que celle des rives des rivières aménagées.

\section{Les autres ministères et organismes publics}

D'autres ministères et organismes peuvent intervenir également dans la gestion des terres publiques selon des modalités propres à chacun d'entre eux, par exemple le ministère de l'Environnement qui est chargé d'assurer la conservation intégrale de certains milieux naturels de grand intérêt en y créant des réserves écologiques.

\section{LES DÉFAuTS DE CE SYSTÈmE}

De nombreux organismes se partagent donc la gestion des terres publiques tant en ce qui concerne le foncier que les ressources naturelles. Leurs attributions n'ont pas toujours des limites bien tracées. II leur arrive même de se chevaucher. La conservation du milieu naturel est par exemple éclatée entre le ministère des Terres et Forêts, le ministère du Tourisme, de la Chasse et de la Pêche et le ministère de l'Environnement. La gestion de l'eau est partagée entre le ministère des Ressources naturelles en ce qui concerne le régime des eaux et le ministère de l'Environnement en

(1) Rappelons que vient d'être créé le Ministère de l'Energie et des Ressources (septembre 1979), peu après la rédaction de cet article. L'organisation et les compétences du nouveau Ministère, qui englobe l'ancien Ministère des Terres et Forêts, n'étaient pas encore connues. 


\section{J. MAZODIER}

ce qui concerne leur qualité. De plus ces ministères disposent de structures administratives souvent insuffisantes et leur action n'est pas suffisamment coordonnée. II en résulte des désordres qui nuisent à la qualité de la gestion du patrimoine.

\section{La connaissance du patrimoine}

Jusqu'à ces dernières années, la tenue du terrier était assurée par le ministère des Terres et Forêts de manière centralisée à Québec, de sorte que ceux qui devaient l'utiliser sur le terrain, y avaient difficilement accès. En outre, les ministères et organismes publics peuvent procéder, si la loi les y autorise, à l'aliénation des territoires placés sous leur juridiction sans en informer nécessairement le ministère des Terres et Forêts. Certains ministères ont même créé des registres officiels, distincts du terrier.

Ces pratiques ont pour conséquence une altération du terrier qui ne décrit plus de manière exacte l'état du territoire public.

Pour tenter de remédier à ces errements, le gouvernement essaye, à titre expérimental, de décentraliser dans certains régions la gestion des documents cadastraux. Cette procédure sera étendue à toutes les régions si le bilan de cette expérience est positif.

En vue de la connaissance des ressources, chaque administration peut procéder a l'inventaire des ressources biologiques, géographiques ou physiques du territoire public pour répondre aux missions qui leur sont confiées. Au cloisonnement de ces missions correspond une connaissance du milieu naturel, lacunaire, hétérogène et dispersée.

\section{La planification de l'utilisation des terres publiques}

L'impression d'immensité inépuisable que donne le territoire public québécois a longtemps conduit l'administration à négliger la planification de son utilisation. La destination des terres publiques était le résultat des politiques de chaque ministère, des pressions économiques, de la demande du public et des interventions du Gouvernement fédéral qui peut exiger les terrains nécessaires à l'exercice de ses missions (parcs nationaux, transports routiers transcanadiens, ports, aéroports, défense, etc.). Aucune autorité ne parvenait à dégager une politique directrice. II n'était pas rare, par exemple, de voir telle décision d'un ministère contrecarrée par un autre ministère. Le ministère des Terres et Forêts qui pourtant tenait une position clé en tant que gestionnaire foncier du territoire public n'était pas en mesure d'assurer cette coordination, son rôle se limitant à satisfaire les besoins au coup par coup.

La mise en valeur de l'espace et de ses ressources se faisait de manière désordonnée, avec un contrôle insuffisant et s'accompagnait souvent de gaspillage. Elle répondait mal à l'intérêt général.

C'est ainsi que les grandes compagnies papetières ont pu obtenir des concessions pour l'exploitation de la ressource ligneuse sur des surfaces considérables, concessions qui leur laissaient la plus grande liberté au détriment souvent du milieu naturel et de la valorisation de la ressource.

Le droit de pêche et de chasse était donné en exclusivité à des clubs privés et ne bénéficiait qu'à une minorité fortunée.

Les sites de grande valeur récréative et touristique (rives des lacs et des rivières) étaient morcelés pour les besoins de la "villégiature" (résidence secondaıre), ce qui les rendait progressivement inaccessibles au public.

De nouveaux territoires étaient ouverts au développement (Côte Nord) ou lacérés par de grandes infrastructures linéaires (autoroutes, couloir d'énergie électrique) sans égard pour les conséquences sur le milieu naturel. 


\section{LE DÉFI}

De telles constatations ont fait prendre conscience au Gouvernement de la nécessité d'une planification de l'utilisation des terres publiques et l'ont conduit à prendre des mesures importantes.

\section{La pêche et la chasse}

Les Québécois recherchent de plus en plus un contact profond avec la nature et désirent profiter de ses bienfaits. Ces aspirations étaient contrariées par la privatisation du droit de pêche et de chasse.

Pour y répondre le Gouvernement a fait un important effort pour ouvrir le territoire au public. A cet effet il a repris aux clubs privés les droits de pêche et de chasse, puis a décidé de confier la gestion des territoires ainsi libérés à des associations locales (zones d'exploitation coordonnée). Les résultats ne furent pas toujours heureux au début. C'est ainsi que l'on a vu certains milieux particulièrement riches tels que des lacs, vidés de leur ressource en quelques mois. On peut espérer cependant que ces associations se montreront responsables et parviendront rapidement à retrouver un bon équilibre. Mais ne risque-t-on pas alors de reconstituer sous une autre forme les clubs?

\section{La villégiature}

Face à la raréfaction des rives des lacs et des rivières accessibles au public à proximité des lieux habités, conséquence de ce que l'on avait, sans compter, satisfait à la demande de privatisation de ces espaces pour les besoins de la villégiature, le Gouvernement a décidé de planifier cette utilisation. Dans ce but, les sites qui se prêtent à cet usage sont inventoriés et font l'objet d'un aménagement global qui réserve une place importante à une utilisation communautaire non privative des rivages. De plus les lots de villégiature ne sont plus vendus mais concédés de façon à ce que l'État conserve la maîtrise foncière des rivages; la personne qui bénéficie de la concession est tenue de l'occuper en respectant un cahier des charges qui règle la qualité de la construction, sa localisation et la conservation de l'environnement forestier.

\section{Le bois}

Les problèmes de l'approvisionnement en bois. la perspective d'un épuisement de la ressource forestière la plus accessible, conduisit le Gouvernement à supprimer progressivement les concessions dont bénéficiaient les compagnies papetières et à leur substituer des plans de gestion. Ces documents précisent les conditions dans lesquelles les compagnies doivent exploiter la ressource forestière. Ils programment les coupes, définissent les équipements routiers nécessaires à la mobilisation du bois et imposent à l'exploitant certaines obligations pour la reconstitution de la forêt et certaines contraintes pour la conservation du milieu naturel (protection des lacs et des cours d'eau).

\section{Les parcs}

Le Gouvernement a décidé la création d'un réseau de parcs provinciaux. Ces parcs tendront à protéger certains territoires représentatifs du patrimoine naturel ou des sites à caractère exceptionnel. Ils seront préservés de tout équipement lourd, de toute coupe commerciale et de toute exploitation minière. Ils seront ouverts au public dans un but éducatif et récréatif.

\section{L'aménagement du territoire et les grandes infrastructures}

Les grands projets d'équipement ou d'aménagement du territoire sont accompagnés d'études de planification écologique et d'études d'impact afin d'en évaluer et d'en minimiser les conséquences 


\section{La forêt au Québec}

pour le milieu naturel (aménagement de la Baie James et de la Rivière Romaine). De plus le Gouvernement envisage de soumettre ces projets à des "audiences publiques ", procédures permettant d'engager une concertation avec le public et d'assurer la surveillance écologique du milieu naturel une fois le projet réalisé.

L'effort accompli ces dernières années pour maîtriser le devenir des terres publiques n'est que l'écho de la volonté politique du Gouvernement du Québec d'utiliser la richesse considérable qu'elles portent en elles pour le bien de la population. Ces terres sont suffisamment vastes pour satisfaire tous les besoins. En raison même de leur dimension, la mise en valeur de ces terres passe probablement par une certaine spécialisation de l'espace contrairement à ce qui se passe en France où la forêt du fait de sa rareté doit répondre à la fois à des objectifs de production, de protection et d'accueil du public. Cette première forme de mise en valeur est en effet plus économique que la seconde. Mais, cette spécialisation ne doit pas conduire à appauvrir les potentialités du patrimoine naturel, par exemple par épuisement des ressources renouvelables ou par rupture de certains équilibres écologiques.

La mise en valeur des terres publiques doit être le résultat d'une démarche de planification de l'espace, tenant compte non seulement des aspirations de la population, mais aussi de la conservation du patrimoine naturel. C'est une décision à caractère politique qui ne peut être prise à bon escient par l'instance compétente que si elle est en mesure d'en évaluer les conséquences. Ceci implique qu'elle puisse disposer de tous les éléments d'information nécessaires, au meilleur coût.

Le Gouvernement provincial du Québec a pris conscience de l'importance de ce problème et des imperfections des réponses apportées par l'organisation politique et administrative actuelle. Aussi a-t-il engagé, depuis plusieurs années déjà, une vaste réflexion pour préciser les responsabilités des niveaux politiques intéressés en matière de mise en valeur des terres publiques, les procédures qu'il convient à cet effet d'appliquer et l'organisation de l'administration chargée de la mise en œuvre de ces politiques, de la gestion des terres publiques et de la police de la ressource.

Josy MAZODIER, Ingénieur du G.R.E.F. Sous-direction de l'Aménagement forestier Service des forêts

\section{BIBLIOGRAPHIE}

QUÉBEC. MINISTĖRE DES TERRES ET FORÊTS. Mars 1977. Proposition de politique de gestion du domaine public foncier. Ministère des Terres et Forêts, Gouv. du Québec. 